\title{
The Characteristics and Survival Outcomes in Patients Aged 70 Years and Older with Nasopharyngeal Carcinoma in the Intensity-Modulated Radiotherapy Era
}

\author{
Ya-Nan Jin, MD ${ }^{1}$ \\ Wang-Jian Zhang, PhD² \\ Xiu-Yu Cai, MD ${ }^{3}$ \\ Mei-Su Li, MD ${ }^{4}$ \\ Wayne R. Lawrence, $\mathrm{PhD}^{2}$ \\ Si-Yang Wang, $\mathrm{MD}^{5}$ \\ Dong-Mei Mai, MD'1 \\ Yu-Yun Du, MD 1 \\ Dong-Hua Luo, MD \\ Hao-Yuan Mo, MD'1
}

*A list author's affiliations appears at the end of the paper.

\section{Purpose}

We aim to examine nasopharyngeal carcinoma (NPC) characteristics and survival outcomes in patients aged 70 years and older in the intensity-modulated radiotherapy (IMRT) era.

\section{Materials and Methods}

From 2006 to 2013, 126 non-metastatic NPC patients aged $\geq 70$ years who were treated with IMRT +/ - chemotherapy were included. Adult Comorbidity Evaluation 27 (ACE-27) was used to measure patient comorbidities. The overall survival (OS) and cancer-specific survival (CSS) were calculated with the Kaplan-Meier method, and differences were compared using the log-rank test. The Cox proportional hazards model was used to carry out multivariate analyses.

\section{Results}

For the entire group, only two patients (1.6\%) presented stage I disease, and up to $84.1 \%$ patients had stage III-IVB disease. All patients had a comorbidity score of 0 in 24 (19.0\%), 1 in 45 (35.7\%), 2 in 42 (33.3\%), and 3 in 15 (11.9\%) patients. The main acute grade during radiotherapy was 3-4 adverse events consisting of mucositis (25.4\%), bone marrow suppression (16.7\%), and dermatitis (8.7\%). After treatment, four patients (3.2\%) developed temporal lobe injury. Five-year CSS and OS rates were 67.3\% (95\% confidence interval [CI], $58.6 \%$ to $77.4 \%)$ and $54.0 \%(95 \% \mathrm{Cl}, 45.6 \%$ to $63.9 \%)$, respectively. Five-year OS was significantly higher for ACE-27 score 0-1 than ACE-27 score 2-3 (72.9\% and 39.9\%, respectively; $p<0.001$ ). Multivariate analyses showed ACE-27 score 0-1 was significantly associated with superior OS (hazard ratio [HR], 3.02; 95\% Cl, 1.64 to 5.55; $p<0.001$ ). In addition, the rate of OS was higher for stage I-III than that of stage IV, with borderline significance (HR, 1.67; 95\% Cl, 0.99 to 2.82; $p=0.053$ ). But no significant advantage was observed in OS when chemotherapy was used ( $p>0.05)$.

\section{Conclusion}

Our findings suggest IMRT +/- chemotherapy has a manageable toxicity and provides an acceptable survival in patients aged $\geq 70$ years with NPC. ACE-27 score was significantly associated with survival outcomes in this group population.

\section{Key words \\ Nasopharyngeal carcinoma, Aged, Characteristics, Survival outcomes, Intensity-modulated radiotherapy}




\section{Introduction}

Nasopharyngeal carcinoma (NPC) disproportionately burdens East and Southeast Asia accounting for two-thirds of new cases worldwide [1]. Among new cases, $40 \%$ are in China, with the highest prevalence in Southern China [1]. The relative risk of NPC increases with age and peaks at approximately 55 years of age and begins to decline at ages $>60$ years [2]. However, the old-age ratio (total number of individuals aged $\geq 65$ years per 100 working age people [20-64 years]) is projected to rise from 13 elderly people per 100 in 2010 to 45 per 100 by 2050 [3]. Moreover, the population of individuals aged 70 years and older are rising from approximately 75 million in 2010 to a projected 225 million by 2050 [3]. The number of elderly patients with NPC will increase with the rapidly growing elderly population in China, and therefore essential to the management of this population, defined in the present study as individuals aged $\geq 70$ years.

Patients ages 70 years and older are often excluded from studies, especially clinical trials due to age exclusion [4,5]. Considering the lack of clinical trials for this group of patients, the treatment for these patients generally follows guidelines tailored for adult patients (age range, 18 to 69 years). Recently, several studies selected patients that were ages 60 to 65 years as a cutoff point for elderly NPC [6-8]. However, this choice may not be completely reasonable, since the physical health among those ages 60-65 years is improving among the general population. In contrast, patients older than 70 years may vastly differ from adults $[9,10]$, for which closer association with multiple comorbidities, poorer performance status, reduced organ reserve, and less social support. For these reasons, the treatment of patients ages 70 years and older warrants further research.

Furthermore, intensity-modulated radiotherapy (IMRT) is slowly replacing two-dimensional conventional radiotherapy (2DRT) as the primary radiotherapy (RT). However, to date, there are no prior studies among patients ages 70 years and older that investigated characteristics and survival outcomes of patients treated with IMRT. Thus, this study aims to fill multiple knowledge gaps by evaluating NPC treatment among patients aged $\geq 70$ years in the IMRT era.

\section{Materials and Methods}

\section{Patient characteristics}

We conducted a retrospective review of case records for patients with NPC treated at Sun Yat-sen University Cancer Center (SYSUCC) from January 2006 to December 2013. Patients included in the analysis were aged $\geq 70$ years, histologically proven NPC, non-metastatic, and treated with IMRT. In all, 126 patients were included in the study. Key raw data was uploaded onto the Research Data Deposit (RDD) public platform (http:// www.researchdata.org.cn), with the approval RDD number as RDDA2017000334 to validate the authenticity of this article.

\section{Clinical staging and co-morbidity assessment}

Patients had undergone pretreatment evaluation comprising of physical examination, hematology and biochemistry profiles, as well as obtained patient complete history information. Additionally, patients also underwent nasopharynx and neck magnetic resonance (MR) imaging, chest X-ray, or computed tomography (CT), CT whole-body bone scan single photon emission, and abdominal ultrasonography. We performed positron emission tomography-computed tomography on 25 of 126 patients (19.8\%). We restaged patients according to guidelines set by the seventh edition of the American Joint Commission on Cancer staging [11]. Moreover, Adult Comorbidity Evaluation 27 (ACE-27) was performed for identifying important medical comorbidities and assess severity among elderly patients [12].

\section{Radiotherapy}

While immobilized in the supine position using a thermoplastic head and shoulder mask, all patients received IMRT. Contrast-enhanced planning CT (3 mm-slice thickness) images from the superior border of frontal sinus to two centimeters below sternoclavicular joint were obtained. Information obtained were transferred to the Monaco treatment planning system (ver. 3.02, Elekta AB, Stockholm, Sweden). The IMRT plan was designed based on previous studies [13], and in accordance with the International Commission on Radiation Units (ICRU) and Measurements Reports 62 [14] and 83 [15].

\section{Chemotherapy and other treatments}

Chemotherapy is required for treatment of locally advanced diseases; however, chemotherapy was not given to some patients due to patient refusal or suggesting intolerance to chemotherapy. The most commonly used regimens for neoadjuvant chemotherapy were cisplatin $\left(80 \mathrm{mg} / \mathrm{m}^{2}\right)$ with docetaxel $\left(80 \mathrm{mg} / \mathrm{m}^{2}\right)$ for every 3 weeks or cisplatin $(80$ $\left.\mathrm{mg} / \mathrm{m}^{2}\right)$ with 5 -fluorouracil $\left(800 \mathrm{mg} / \mathrm{m}^{2} /\right.$ day over 120 hours). Concurrent chemotherapy consisted of cisplatin (80 or $100 \mathrm{mg} / \mathrm{m}^{2}$ ) on weeks 1,4 , and 7 of radiotherapy, or cis- 
platin $\left(40 \mathrm{mg} / \mathrm{m}^{2}\right)$ weekly. Modification of dosage was performed, if necessary, at the doctors' discretion. In addition, there were patients that received other therapies besides chemotherapy $(n=11)$. Seven patients underwent targeted therapy concurrent with IMRT, consisting of two by cetuximab and five by nimotuzumab. Another four patients received primary tumor brachytherapy with CT-guided permanent implantation of ${ }^{125}$ I seeds after IMRT.

\section{Image assessment of temporal lobe injury}

In the present study, a diagnosis of temporal lobe injury (TLI) was based on follow-up MR images. We must also note that the diagnosis of TLI was based on the criteria described by Wang et al. [16]. The criteria used were as follow: (1) white matter lesions were defined as areas of finger-like lesions of increased signal intensity on T2-weighted images in the temporal lobe; (2) contrast-enhanced lesions were defined as lesions with or without necrosis on post-contrast T1-weighted images with heterogeneous signal abnormalities on T2-weighted images; and (3) determined cysts as round or oval well-defined lesions of very high signal intensity on T2-weighted images with a thin or imperceptible wall.

\section{Follow-up and statistical analysis}

During IMRT, we evaluated patients at least once a week. After 1 month of RT completion, the first assessment of response to treatment was performed. Afterwards, every 3 months during the first 3 years patient evaluation was performed. Thereafter, every 6 months for the following 2 years, and annually afterwards. We measured survival times as the initial start of RT to last follow-up visit or date of the event. Overall survival (OS) was measured as the period from first RT treatment to the last follow-up visit or date of death. We defined cancer-specific survival (CSS) as the period from initial RT treatment to the date of death as a result of NPC or the last follow-up visit.

Kaplan-Meier method was used to calculate actuarial rates, and the differences were compared using the log-rank test. Covariates including age, sex, ACE-27 score, N category, T category, overall stage, radiation dose, and chemotherapy were included in all tests. Cox proportional hazards model was performed to carry out multivariate analyses. p-value of $<0.05$ was determine statistically significant. $R$ software (ver. 3.2.3) was used to perform all statistical analyses.

\section{Ethical statement}

The present study was approved by the Institutional Review Board at SYUCC and conducted in compliance with institutional policy to protect patients' private information.
Table 1. Patient and tumor characteristics

\begin{tabular}{|c|c|}
\hline Characteristic & $\begin{array}{l}\text { No. of patients }(\%) \\
\qquad(\mathrm{n}=126)\end{array}$ \\
\hline Age, median (range, yr) & $73(70-80)$ \\
\hline \multicolumn{2}{|l|}{ Sex } \\
\hline Male & $96(76.2)$ \\
\hline Female & $30(23.8)$ \\
\hline \multicolumn{2}{|l|}{ ACE-27 score } \\
\hline 0 & $24(19.0)$ \\
\hline 1 & $45(35.7)$ \\
\hline 2 & $42(33.3)$ \\
\hline 3 & $15(11.9)$ \\
\hline \multicolumn{2}{|l|}{ T category ${ }^{a)}$} \\
\hline $\mathrm{T} 1$ & $7(5.6)$ \\
\hline $\mathrm{T} 2$ & $20(15.9)$ \\
\hline $\mathrm{T} 3$ & $59(46.8)$ \\
\hline $\mathrm{T} 4$ & $40(31.7)$ \\
\hline \multicolumn{2}{|l|}{$\mathrm{N}$ category ${ }^{\mathrm{a})}$} \\
\hline N0 & $16(12.7)$ \\
\hline N1 & $61(48.4)$ \\
\hline N2 & $37(29.4)$ \\
\hline N3 & $12(9.5)$ \\
\hline \multicolumn{2}{|l|}{ Overall stage $\mathrm{e}^{\mathrm{a})}$} \\
\hline I & $2(1.6)$ \\
\hline II & $18(14.3)$ \\
\hline III & $60(47.6)$ \\
\hline IVA & $37(29.4)$ \\
\hline IVB & $9(7.1)$ \\
\hline \multicolumn{2}{|l|}{ Radiation dose (Gy) } \\
\hline$\leq 68$ (range, 64-68) & $57(45.2)$ \\
\hline$>68$ (range, 68-73) & $69(54.8)$ \\
\hline \multicolumn{2}{|l|}{ Chemotherapy } \\
\hline No chemotherapy & $72(57.1)$ \\
\hline Concurrent chemoradiotherapy & $42(33.3)$ \\
\hline Neoadjuvant+concurrent chemoradiotherapy & py $12(9.5)$ \\
\hline
\end{tabular}

ACE-27, Adult Comorbidity Evaluation 27. a)The 7th American Joint Commission on Cancer staging system.

\section{Results}

\section{Symptoms, signs, and comorbidity before treatment}

Table 1 presents patients' clinical characteristics. Median age at diagnosis was 73 years (ages ranged 70 to 80 years). Among the 126 patients, only two patients (1.6\%) had stage I disease, and $84.1 \%$ patients presented III/IVA-B disease. The most common symptom was cervical mass with an incidence of $52 \%$ (66 patients), followed by nasal problems (55 patients, $44 \%$ ), auditory problems (46 patients, 37\%), and headache (22 patients, 17\%). The ACE-27 score for comorbid- 


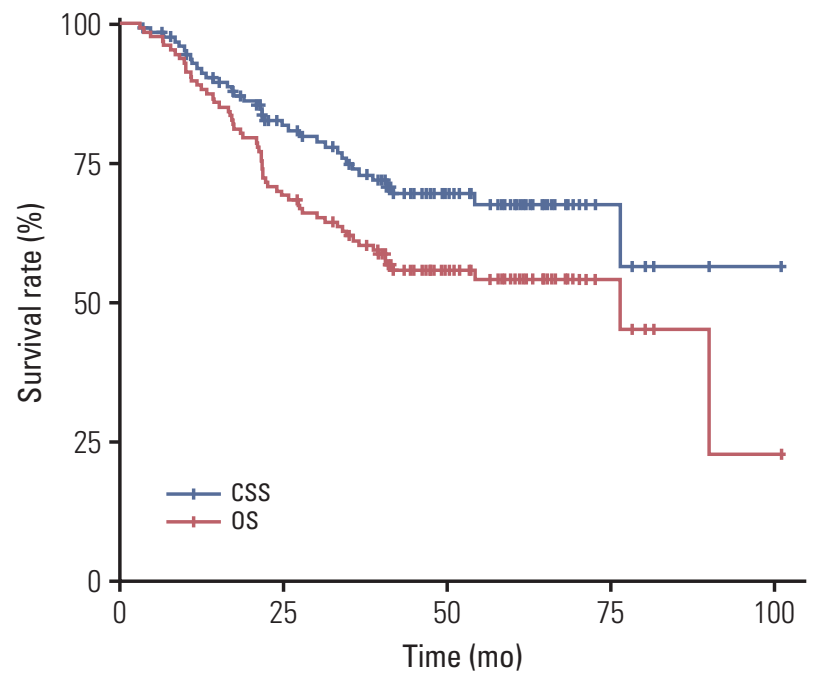

Fig. 1. Survival rate for patients aged $\geq 70$ years treated with intensity-modulated radiotherapy +/ - chemotherapy. CSS, cancer-specific survival; OS, overall survival.

ity was 0 in $24(19.0 \%), 1$ in $45(35.7 \%), 2$ in $42(33.3 \%)$, and 3 in $15(11.9 \%)$.

\section{Treatment course and toxicity}

All patients completed the planned RT. The median radiation dose for primary tumor and regional lymph node were 68.4 Gy (range, 64.2 to 73.4 Gy) and 64.6 Gy (range, 59.6 to $68.4 \mathrm{~Gy})$, respectively. Overall, 72 patients $(57 \%)$ were treated with RT alone, 42 patients $(33 \%)$ received concurrent chemoradiation therapy (CCRT), and 12 patients (10\%) received neoadjuvant chemotherapy plus CCRT. Mucositis, bone marrow suppression, and dermatitis were the most common acute toxicities where majority developed within grade 1 to 2 , and the incidence of grades 3 to 4 acute toxicities were $18.3 \%, 10.3 \%$, and $6.3 \%$, respectively. During follow-up, four patients $(3.2 \%)$ developed radiation-induced TLI. Of the four patients, two patients were staged with T4 disease, the other two patients with T3 disease, and no patients with T1-2 disease developed TLI.

\section{Prognostic factors and survival}

Following a median follow-up of 40.6 months (range, 3 to 101 months), 58 (46\%) patients died. Among the patients that died, $36(62.1 \%)$ died from NPC, $5(8.6 \%)$ died of toxicities related to treatment, and $17(29.3 \%)$ died from internal medical diseases that were unrelated to cancer. Of the 17 patients who died because of internal medical disease, eight were chest infection, six cerebral vascular accidents, one because of second malignancies, and two due to other specific causes. Kaplan-Meier analysis of OS and CSS are presented in Fig. 1. Estimated OS and CSS rates at 5 years were $54.0 \%$ (95\% confidence interval [CI], $45.6 \%$ to $63.9 \%$ ) and $67.3 \%$ (95\% CI, $58.6 \%$ to $77.4 \%$ ), respectively. ACE-27 score $0-1$ was associated with higher 5-year OS in comparison with that of ACE-27 score 0-1 (72.9\% and 39.9\%, respectively; $\mathrm{p}<0.001)$ (Fig. 2A). The OS was significantly elevated for stage I-III than stage IV $(62.1 \%$ and $40.1 \%$, respectively; $\mathrm{p}=0.018)$ (Fig. 2C). However, we failed to confirm the positive association of chemotherapy (hazard ratio [HR], 1.30; 95\% CI, 0.77 to $2.20 ; \mathrm{p}=0.318$ ) (Fig. 2B) and radiation dose (HR, 1.00; 95\% CI, 0.59 to $1.70 ; p=0.999$ ) (Fig. 2D) with OS. Utilizing the Cox proportional hazards model, we observed the ACE-27 score 0-1 was significantly associated with superior OS (HR, 3.02; 95\% CI, 1.64 to 5.55; $\mathrm{p}<0.001$ ), and stage IV was associated with inferior OS, with borderline significance (HR, 1.67; 95\% CI, 0.99 to 2.82; $\mathrm{p}=0.053$ ) (Table 2).

\section{Prognostic value of chemotherapy in subgroups of NPC patients}

Chemotherapy prognostic significance in patients with stage III-IV disease were analyzed. However, stage III-IV diseases did not present a unique patient subgroup, where the addition of chemotherapy to RT was efficacious in improving 5-year OS (56.6\% vs. 51.2\%, p=0.617) (Fig. 3A). We further analyzed the prognostic value of chemotherapy in the subgroups of patients with NPC with different ACE-27 score. Among patients with ACE-27 score $<2$, though the 5-year OS rates $(78.0 \%$ vs. $69.1 \%, \mathrm{p}=0.554)$ (Fig. 3B) were higher in patients treated with chemo-radiotherapy than RT alone, this did not reach statistical significance. In contrast, compared with RT alone, chemo-radiotherapy was associated with a trend towards a lower OS for patients with ACE-27 score $\geq 2$, with borderline association ( $50.3 \%$ vs. $26.4 \%, \mathrm{p}=0.071$ ) (Fig. 3C).

\section{Discussion}

To the best of our knowledge, this is the first study on clinical outcomes of patients aged $\geq 70$ years with NPC treated by IMRT. The 5-year OS and CSS rates were $54.0 \%$ and $67.3 \%$, respectively. Our findings suggest that IMRT +/chemotherapy provided promising long-term survival in patients aged $\geq 70$ years with NPC. Multivariate analyses showed that ACE-27 score 0-1 was significantly associated with superior OS. Additionally, a significant delay in the diagnosis of NPC may exist for patients aged $\geq 70$ years, as 
A
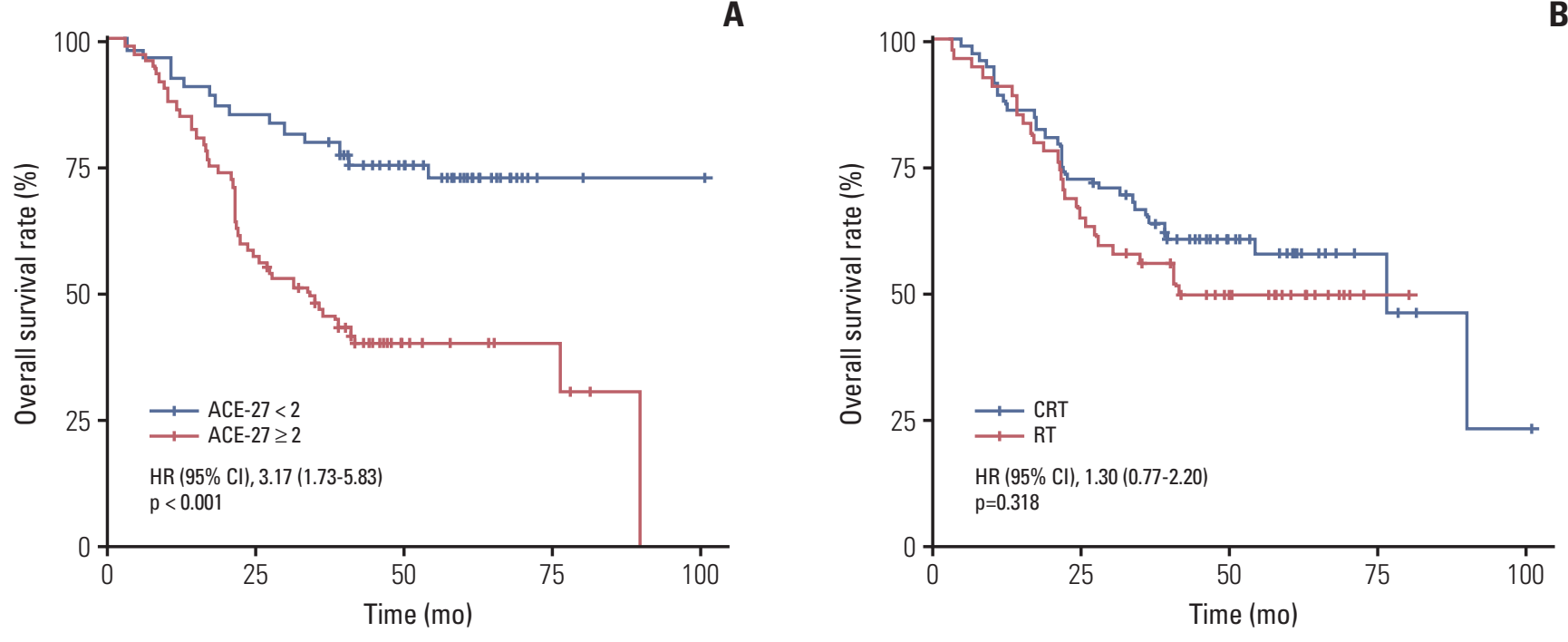

C
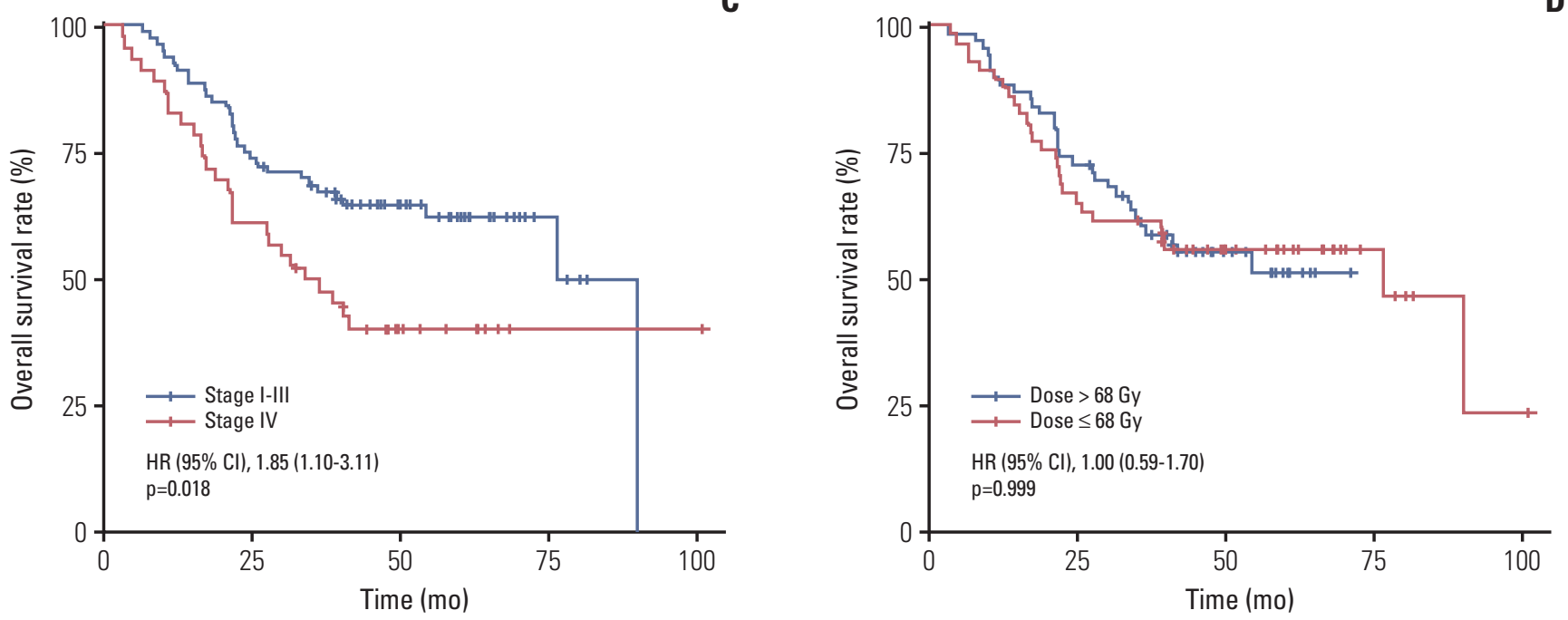

Fig. 2. Kaplan-Meier analysis of overall survival is stratified by ACE-27 (A), chemotherapy (B), overall stage (C), and primary tumor dose (D). HR, hazard ratio; CI, confidence interval; ACE-27, Adult Comorbidity Evaluation 27; CRT, chemoradiotherapy; RT, radiotherapy.

approximately $84 \%$ of elderly NPC patients had stage III-IV disease at presentation, much higher than $70 \%$ among adult age group [17-19].

In the current study, our results suggested acceptable survival (5-year OS, $54.0 \%$ ) in patients aged $\geq 70$ years. Recently, a study by Sze et al. [9] found that the 5-year OS rate was $43.9 \%$ among patients aged $\geq 70$ years. This inconsistency might be due to the application of IMRT in our study, which has shown to produce exceptional treatment results compared with 2DRT $[20,21]$, but only $29.1 \%$ of patients in the study of Sze et al. [9] received IMRT. Additionally, consid- ering the performance status of patients aged $\geq 70$ years varying greatly, the heterogeneity of patients included in the study by Sze et al. [9] and the present study might contribute to the inconsistency of treatment outcomes. Results of our study demonstrated that ACE-27 was the strongest prognostic factor for OS in elderly patients aged $\geq 70$ years. Considering that patients aged $\geq 70$ years are commonly in close association with multiple comorbidities and poor performance status, which effect treatment outcomes, the use of ACE-27 score in the evaluation of elderly patients is necessary in the present study. In fact, the association between sur- 
Table 2. Univariate and multivariate analysis of prognostic factors for overall survival

\begin{tabular}{|c|c|c|c|c|}
\hline \multirow{2}{*}{ Variable } & \multicolumn{2}{|c|}{ Univariate analysis } & \multicolumn{2}{|c|}{ Multivariate analysis } \\
\hline & HR $(95 \%$ CI $)$ & p-value & HR $(95 \% \mathrm{CI})$ & p-value \\
\hline Age $(<75$ yr vs. $\geq 75$ yr $)$ & $0.73(0.36-1.50)$ & 0.398 & NS & NS \\
\hline Sex (male vs. female) & $1.11(0.60-2.06)$ & 0.746 & NS & NS \\
\hline T category ${ }^{\mathrm{a})}$ (T1-2 vs. T3-4) & $1.36(0.69-2.70)$ & 0.371 & NS & NS \\
\hline $\mathrm{N}$ category ${ }^{\text {a) }}$ (N0-1 vs. N2-3) & $1.67(1.15-2.89)$ & 0.034 & $1.37(0.87-3.12)$ & 0.067 \\
\hline Overall stage ${ }^{a)}(\mathrm{I}-\mathrm{III}$ vs. IV) & $1.85(1.10-3.11)$ & 0.020 & $1.67(0.99-2.82)$ & 0.053 \\
\hline Radiation dose ( $\leq 68$ Gy vs. $>68$ Gy) & $1.00(0.59-1.70)$ & 0.999 & NS & NS \\
\hline Chemotherapy (no vs. yes) & $1.30(0.77-2.20)$ & 0.318 & NS & NS \\
\hline ACE-27 score $(<2$ vs. $\geq 2)$ & $3.17(1.73-5.83)$ & $<0.001$ & $3.02(1.64-5.55)$ & $<0.001$ \\
\hline
\end{tabular}

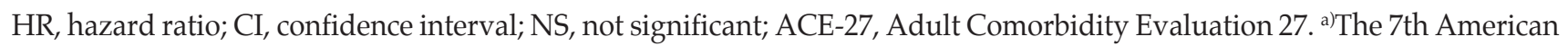
Joint Commission on Cancer staging system.

vival and ACE-27 has been demonstrated in a wide range of different cancers [22], and specifically in head and neck cancers [12,23]. Rogers et al. [23] evaluated the prognostic value of ACE-27 on head and neck cancer patients, and suggested that ACE-27 was robustly correlated with survival. Paleri et al. [24] even found that moderate and severe comorbidity evaluated using ACE-27 had a greater impact on survival than TNM stage in laryngeal squamous cancer patients. In contrast, Ramakrishnan et al. [25] reported that ACE-27 had no adverse role on 59 NPC patients' prognosis. This inconsistency might be a result of small sample size, as noted by the authors.

Given the limited number of studies, the efficacy of chemotherapy in elderly patients with NPC remains unclear. Two prior studies [6,7] demonstrated that chemotherapy was associated with superior OS in elderly NPC patients after 2DRT. In contrast, our results failed to confirm the role of chemotherapy as a prognostic factor in elderly patients treated with IMRT. Considering that IMRT was adopted in the current study, this seems to be reasonable as patients had improved survival outcomes after IMRT [20,21], by which the actual benefit of chemotherapy might be diluted for this group. However, it should be mentioned that potential selection bias was hard to avoid in the use of chemotherapy in the present study. Further propensity-matched study or even randomized clinical trial are still needed to confirm the effect of chemotherapy in patients ages $\geq 70$ years treated by IMRT.

In respect to previous studies, approximately $70 \%$ of NPC adult patients are diagnosed with stage III/IVA-B [16-18]. While we found higher proportion $(83.3 \%)$ of patients with stage III/IVA-B in the current study, only two patients (1.6\%) had stage I disease. Additionally, several studies $[19,26]$ reported that among adult patients with NPC, approximately $40 \%$ presented with cervical mass. However, in the current study, cervical mass was present at diagnosis in 50\% of patients. This suggests elderly patients who develop NPC may not have received prompt diagnosis and evaluation. Delayed diagnosis may occur in patients aged 70 years and older because of easily overlooked symptoms such as rhinobyon, headache, and tinnitus. Thus, for elderly patients, physicians should be more aggressive in the workup and diagnostic evaluation of NPC symptoms, as well as provide timely biopsies and nasopharyngoscope exams if indicated.

TLI is a serious late sequela following radical RT of NPC and associated with severe impairment of quality of life and survival [27]. Temporal lobe protection is arduous, particularly in patients with T3-4 disease that may have extensive skull base invasion or cavernous sinus involvement. Reported rates of TLI range from $7.5 \%$ to $12.9 \%$ in NPC patients after IMRT [28-30]. Given the high proportion of T34 disease (79\%) in the current series, TLI incidence is expected to increase. However, in the present study, the incidence of $3.2 \%$ is lower than reported in prior studies. Moreover, given the poor survival among NPC patients older than 70 years $[8,9]$, findings that they did not show a high incidence of TLI was probably due to a high proportion may have died prior to TLI could have develop. Therefore, the incidence of TLI in elderly patients may have been underestimated in the present study.

One major limitation in the present study is the small patient sample size and the influence this may have had on identifying an effect. However, the low NPC incidence in elderly patients must be noted, particularly those aged $\geq 70$ years. Another limitation is that our study was limited by the retrospective nature, and the possible selection bias of using chemotherapy for patients was difficult to avoid. Additionally, a third concern was the lack on inclusion of data about pretreatment Epstein-Barr Virus DNA concentrations, which has shown to strongly predict NPC survival [31-33]. Further 
A

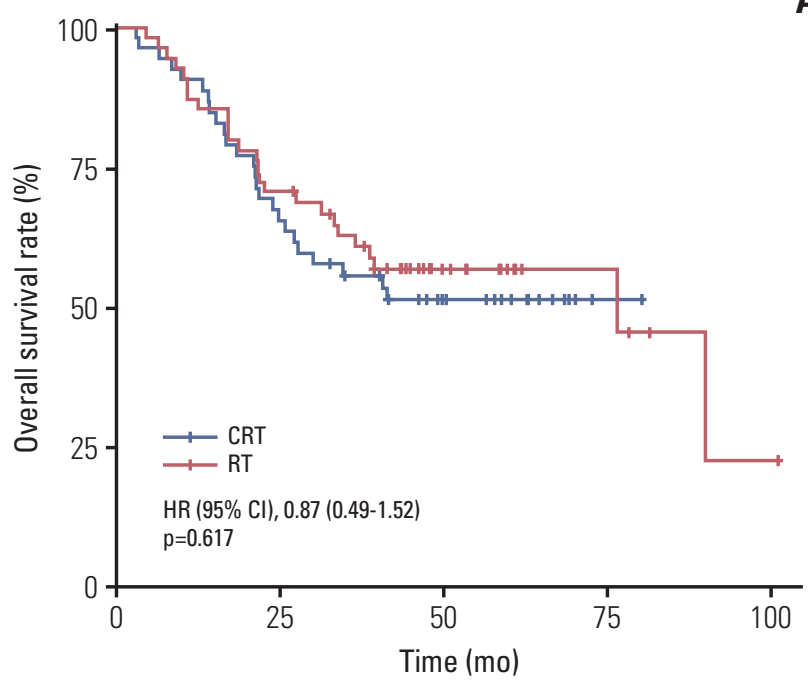

C
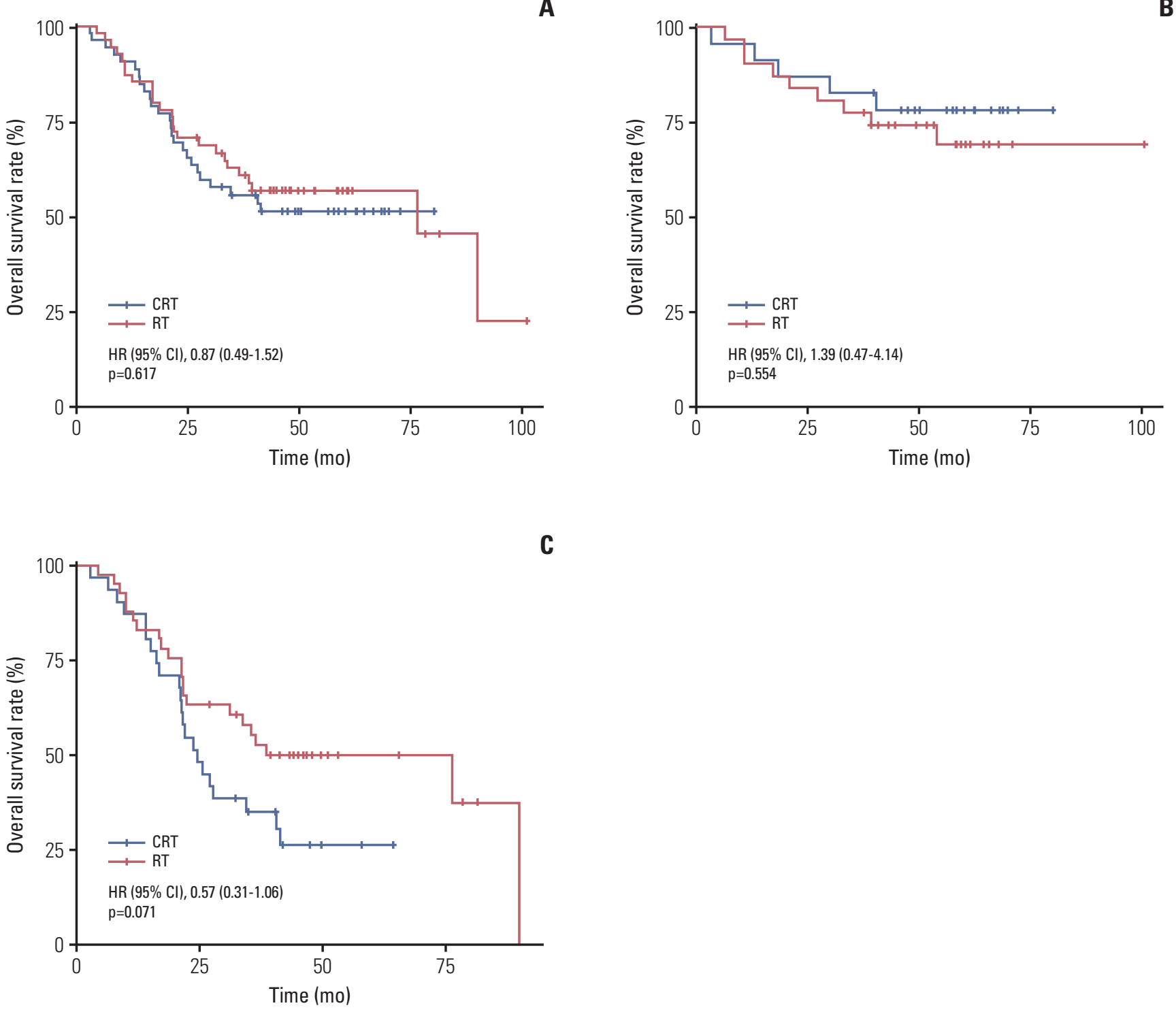

Fig. 3. Kaplan-Meier analysis of overall survival stratified by chemotherapy (RT vs. CRT) in patients with locoregionally advanced nasopharyngeal carcinoma (A), ACE-27 score $<2$ (B), and ACE-27 score $\geq 2$ (C). HR, hazard ratio; CI, confidence interval; RT, radiotherapy; CRT, chemoradiotherapy; ACE-27, Adult Comorbidity Evaluation 27.

studies (e.g., propensity-matched study) are urgently needed to classify subgroups that would benefit most from chemotherapy, since not all patients aged $\geq 70$ years can benefit from chemotherapy.

In summary, our results suggest that IMRT + / - chemotherapy has a manageable toxicity and provides an acceptable survival in patients aged $\geq 70$ years. We observed a significant association between ACE-27 score with survival outcomes in this group of patients. Although we failed to confirm the benefit of chemotherapy for this group of patients, further studies are urgently needed to classify the subgroup who could gain most from chemotherapy.

\section{Conflicts of Interest}

Conflict of interest relevant to this article was not reported.

\section{Acknowledgments}

The present study was funded by the National Science \& Technology Pillar Program during the Twelfth Five-year Plan Period (No.2014BAI09B10). 


\section{Author Details}

${ }^{1}$ State Key Laboratory of Oncology in Southern China, Collaborative Innovation Center of Cancer Medicine, Department of Nasopharyngeal Carcinoma, Sun Yat-sen University Cancer Center, Guangzhou, ${ }^{2}$ Department of Medical Statistics and Epidemiology and Health Information Research Center and Guangdong Key Laboratory of Medicine, School of Public Health, Sun Yat-sen University, Guang- zhou, ${ }^{3}$ State Key Laboratory of Oncology in Southern China, Collaborative Innovation Center of Cancer Medicine, Department of VIP Region, Sun Yat-sen University Cancer Center, Guangzhou, ${ }^{4}$ Department of Medical Records Statistics, The Fifth Affiliated Hospital of Sun Yat-sen University, Zhuhai, ${ }^{5}$ Department of Radiation Oncology, The Fifth Affiliated Hospital of Sun Yat-sen University, Zhuhai, China

\section{References}

1. Chua ML, Wee JT, Hui EP, Chan AT. Nasopharyngeal carcinoma. Lancet. 2016;387:1012-24.

2. Zhang LF, Li YH, Xie SH, Ling W, Chen SH, Liu Q, et al. Incidence trend of nasopharyngeal carcinoma from 1987 to 2011 in Sihui County, Guangdong Province, South China: an ageperiod-cohort analysis. Chin J Cancer. 2015;34:350-7.

3. United Nations. World population prospects: the 2010 revision. New York: United Nations; 2011.

4. Hui EP, Ma BB, Leung SF, King AD, Mo F, Kam MK, et al. Randomized phase II trial of concurrent cisplatin-radiotherapy with or without neoadjuvant docetaxel and cisplatin in advanced nasopharyngeal carcinoma. J Clin Oncol. 2009;27: 242-9.

5. Chen L, Hu CS, Chen XZ, Hu GQ, Cheng ZB, Sun Y, et al. Concurrent chemoradiotherapy plus adjuvant chemotherapy versus concurrent chemoradiotherapy alone in patients with locoregionally advanced nasopharyngeal carcinoma: a phase 3 multicentre randomised controlled trial. Lancet Oncol. 2012;13:163-71.

6. Liu H, Chen QY, Guo L, Tang LQ, Mo HY, Zhong ZL, et al. Feasibility and efficacy of chemoradiotherapy for elderly patients with locoregionally advanced nasopharyngeal carcinoma: results from a matched cohort analysis. Radiat Oncol. 2013;8:70.

7. Zeng Q, Xiang YQ, Wu PH, Lv X, Qian CN, Guo X. A matched cohort study of standard chemo-radiotherapy versus radiotherapy alone in elderly nasopharyngeal carcinoma patients. PLoS One. 2015;10:e0119593.

8. Zhang Y, Yi JL, Huang XD, Xu GZ, Xiao JP, Li SY, et al. Inherently poor survival of elderly patients with nasopharyngeal carcinoma. Head Neck. 2015;37:771-6.

9. Sze HC, Ng WT, Chan OS, Shum TC, Chan LL, Lee AW. Radical radiotherapy for nasopharyngeal carcinoma in elderly patients: the importance of co-morbidity assessment. Oral Oncol. 2012;48:162-7.

10. Xiao G, Cao Y, Qiu X, Wang W, Wang Y. Influence of gender and age on the survival of patients with nasopharyngeal carcinoma. BMC Cancer. 2013;13:226.

11. Edge SB, Compton CC. The American Joint Committee on Cancer: the 7th edition of the AJCC cancer staging manual and the future of TNM. Ann Surg Oncol. 2010;17:1471-4.
12. Piccirillo JF. Importance of comorbidity in head and neck cancer. Laryngoscope. 2000;110:593-602.

13. Lai SZ, Li WF, Chen L, Luo W, Chen YY, Liu LZ, et al. How does intensity-modulated radiotherapy versus conventional two-dimensional radiotherapy influence the treatment results in nasopharyngeal carcinoma patients? Int J Radiat Oncol Biol Phys. 2011;80:661-8.

14. ICRU report. Prescribing, recording, and reporting photon beam therapy (Report 62). Bethesda, MD: International Commission on Radiation Units and Measurements; 1999.

15. ICRU report. Prescribing, recording, and reporting photonbeam intensity-modulated radiation therapy (IMRT) (Vol. 83). Bethesda, MD: International Commission on Radiation Units and Measurements; 2010

16. Wang YX, King AD, Zhou H, Leung SF, Abrigo J, Chan YL, et al. Evolution of radiation-induced brain injury: MR imagingbased study. Radiology. 2010;254:210-8.

17. Guo R, Sun Y, Yu XL, Yin WJ, Li WF, Chen YY, et al. Is primary tumor volume still a prognostic factor in intensity modulated radiation therapy for nasopharyngeal carcinoma? Radiother Oncol. 2012;104:294-9.

18. Li WF, Sun Y, Chen M, Tang LL, Liu LZ, Mao YP, et al. Locoregional extension patterns of nasopharyngeal carcinoma and suggestions for clinical target volume delineation. Chin J Cancer. 2012;31:579-87.

19. Yi JL, Gao L, Huang XD, Li SY, Luo JW, Cai WM, et al. Nasopharyngeal carcinoma treated by radical radiotherapy alone: ten-year experience of a single institution. Int J Radiat Oncol Biol Phys. 2006;65:161-8.

20. Zhang MX, Li J, Shen GP, Zou X, Xu JJ, Jiang R, et al. Intensity-modulated radiotherapy prolongs the survival of patients with nasopharyngeal carcinoma compared with conventional two-dimensional radiotherapy: a 10-year experience with a large cohort and long follow-up. Eur J Cancer. 2015;51:258795.

21. Peng G, Wang T, Yang KY, Zhang S, Zhang T, Li Q, et al. A prospective, randomized study comparing outcomes and toxicities of intensity-modulated radiotherapy vs. conventional two-dimensional radiotherapy for the treatment of nasopharyngeal carcinoma. Radiother Oncol. 2012;104:286-93.

22. Piccirillo JF, Tierney RM, Costas I, Grove L, Spitznagel EL Jr. 
Prognostic importance of comorbidity in a hospital-based cancer registry. JAMA. 2004;291:2441-7.

23. Rogers SN, Aziz A, Lowe D, Husband DJ. Feasibility study of the retrospective use of the Adult Comorbidity Evaluation index (ACE-27) in patients with cancer of the head and neck who had radiotherapy. Br J Oral Maxillofac Surg. 2006;44: 283-8.

24. Paleri V, Wight RG, Davies GR. Impact of comorbidity on the outcome of laryngeal squamous cancer. Head Neck. 2003;25: 1019-26.

25. Ramakrishnan Y, Paleri V, Shah R, Steen IN, Wight RG, Kelly CG. Comorbidity in nasopharyngeal carcinoma: a preliminary communication on the prevalence, descriptive distribution and impact on outcome. Clin Otolaryngol. 2007;32:484-8.

26. Lee AW, Foo W, Law SC, Poon YF, Sze WM, O SK, et al. Nasopharyngeal carcinoma: presenting symptoms and duration before diagnosis. Hong Kong Med J. 1997;3:355-61.

27. Cheung MC, Chan AS, Law SC, Chan JH, Tse VK. Impact of radionecrosis on cognitive dysfunction in patients after radiotherapy for nasopharyngeal carcinoma. Cancer. 2003;97: 2019-26.

28. Zhou GQ, Yu XL, Chen M, Guo R, Lei Y, Sun Y, et al. Radiation-induced temporal lobe injury for nasopharyngeal carcinoma: a comparison of intensity-modulated radiotherapy and conventional two-dimensional radiotherapy. PLoS One. 2013;8:e67488

29. Zeng L, Tian YM, Sun XM, Chen CY, Han F, Xiao WW, et al. Late toxicities after intensity-modulated radiotherapy for nasopharyngeal carcinoma: patient and treatment-related risk factors. Br J Cancer. 2014;110:49-54.

30. Kong C, Zhu XZ, Lee TF, Feng PB, Xu JH, Qian PD, et al. LASSO-based NTCP model for radiation-induced temporal lobe injury developing after intensity-modulated radiotherapy of nasopharyngeal carcinoma. Sci Rep. 2016;6:26378.

31. Bortolin MT, Pratesi C, Dolcetti R, Bidoli E, Vaccher E, Zanussi $S$, et al. Clinical value of Epstein-Barr virus DNA levels in peripheral blood samples of Italian patients with undifferentiated carcinoma of nasopharyngeal type. Cancer Lett. 2006; 233:247-54.

32. Lin JC, Wang WY, Liang WM, Chou HY, Jan JS, Jiang RS, et al. Long-term prognostic effects of plasma epstein-barr virus DNA by minor groove binder-probe real-time quantitative PCR on nasopharyngeal carcinoma patients receiving concurrent chemoradiotherapy. Int J Radiat Oncol Biol Phys. 2007; 68:1342-8.

33. Tang LQ, Chen QY, Guo SS, Chen WH, Li CF, Zhang L, et al. The impact of plasma Epstein-Barr virus DNA and fibrinogen on nasopharyngeal carcinoma prognosis: an observational study. Br J Cancer. 2014;111:1102-11. 